\title{
Psychoanalytische Konzepte der Psychosentherapie
}

\author{
Frank Schwarz
}

Online publiziert: 8. August 2012

(C) Springer-Verlag 2012

Es ist mittlerweile über hundert Jahre her, dass Freud mit seiner Interpretation der Autobiografie Schrebers einen Meilenstein gesetzt hat. Auch wenn es sich dabei nicht um seine erste Beschäftigung mit diesem Thema handelte, mag ihr wegen des immensen Interesses, das sie auslöste, diese Position eingeräumt werden. Daraus konnte zwar keine allgemeine psychodynamische Theorie der Psychosen entwickelt werden, aber die psychoanalytische Annäherung an dieses Thema ist damit erstmals ausführlich unternommen worden, und zwar mit der stimmigen Annahme, dass es möglich sein müsste, Verbindungen zwischen der psychotischen Symptombildung und der Lebensgeschichte herzustellen, ähnlich wie dies bei der Traumdeutung geschieht.

Seit diesem Beginn durch Freud ist es zunächst mit der Entwicklung der Psychosenpsychotherapie nur schleppend vorangegangen, und es hat bis zu einem systematischen internationalen Austausch über die psychoanalytisch begründete Arbeit mit psychotischen Patienten recht lange gedauert. Erst 1956 wurde die International Society for the Psychological Treatments of Schizophrenias and other Psychoses (ISPS), in der auch die psychoanalytisch arbeitenden Therapeuten repräsentiert sind, gegründet, und seitdem finden alle drei bis vier Jahre internationale Kongresse dieser Gesellschaft statt. Im vergangenen Jahr kam es sogar zur Gründung einer deutschen Gruppe (ISPS Germany). Bei Alanen et al. (2009) findet sich eine profunde Übersicht über die Entwicklung der ISPS bzw. der Geschichte der Psychosenpsychotherapie, einschließlich der im deutschsprachigen Raum. Hier werden auch detailliert die verschiedenen Aktivitäten in Deutschland aufgeführt. Eine davon ist die vor zwanzig Jahren begonnene von München ausgehende Initiative mit dem Ziel, auf dem Gebiet der Psychosenpsychotherapie arbeitende Psychoanalytiker zu einem regelmäßigen Austausch anzuregen und gleichzeitig eine Fort- und Weiterbildung anzubieten

Dr. med. F. Schwarz $(\bowtie)$

Elisabethstr. 44, 80796 München, Deutschland

E-Mail: FHSchwarz@t-online.de 
(überregionale Weiterbildung in analytischer Psychosentherapie). Eine ausführliche Beschreibung findet sich bei Schwarz und Dümpelmann 2011. In den vergangenen zwei Jahrzehnten kam es auf diese Weise zu einer intensiven Kooperation von zahlreichen Experten auf dem Gebiet der Psychosenpsychotherapie, die zu dem angestrebten Ziel einer Verbesserung und Differenzierung der therapeutischen Möglichkeiten führte.

Die Autoren der Schwerpunktbeiträge in diesem Heft des Forum der Psychoanalyse befinden sich in diesem Sinne in einem langjährigen Austauschprozess. Sie haben alle reichhaltige Erfahrung in der analytischen Psychotherapie von psychotischen Patienten und können ihre jeweiligen theoretischen Positionen durch die Reflexion der von ihnen durchgeführten Behandlungen überprüfen und ggf. verändern. Ihre Arbeiten beleuchten von verschiedenen Seiten her das komplexe Feld der psychoanalytischen Beschäftigung mit Psychosen, wobei jeweils differenzierte Falldarstellungen dazu dienen, die diskutierte Problematik zu veranschaulichen. In dem Beitrag von Matejek werden behandlungstechnische Gesichtspunkte subtil dargestellt und hinterfragt, sodass konkrete Vorgehensweisen in den einzelnen Sitzungen nachvollzogen werden können. Übertragungs- und Gegenübertragungsvorgänge können durch die plastischen Schilderungen quasi miterlebt werden. Dies gilt insbesondere auch für vorsprachliche Beziehungsaspekte. Der Beitrag von Bruns erscheint insofern besonders aktuell, als er sich mit den psychodynamischen Zusammenhängen zwischen traumatischen Erfahrungen und späteren selbstschädigenden Reinszenierungen dieser Erlebnisse bei psychotischen Patienten auseinandersetzt. Er führt auch aus, wie institutionelle Bedingungen an solchen Vorgängen beteiligt sein können. Dieser Ansatz ist auch deshalb von Bedeutung, weil er über die bisherigen oft sehr hypothetischen Ansätze über die frühe Psychogenese psychotischer Störungen hinausgeht und sich um tatsächlich stattgefundene schädigende Ereignisse verstärkt kümmert. Schwarz geht vom Fall Schreber aus, bei dem erstmals Zusammenhänge zwischen psychotischen und biografischen Inhalten aufzuzeigen versucht wurden, wobei sich erst Jahrzehnte später durch eine Forschung, die sich mit Schrebers Vater befasste, herausstellte, dass es offensichtlich schwere intrafamiliäre Traumata gegeben haben musste. Anhand von zwei Langzeitbehandlungen wird veranschaulicht, wie psychotische Symptome lebensgeschichtlich bedingte und aktuell zugespitzte Konflikte in einer Art und Weise darstellen, die einem psychodynamischen Verständnis zugänglich ist, was auch für die eine Psychose auslösenden Anlässe gilt.

\section{Literatur}

Alanen YO, González de Chavez M, Silver A-LS, Martindale B (2009) (Hrsg) Psychotherapeutic approaches to schizophrenic psychoses. Routledge, London, S 23-37

Schwarz F, Dümpelmann M (2011) München 1992. Weg zu einer integrierenden Psychosenpsychotherapie. Aufbruch zu Kooperation und gemeinsamer Identität. In: Haebler D von, Mentzos S, Lempa G (Hrsg) Psychosenpsychotherapie im Dialog. Zur Gründung des DDPP. Forum Psychoanal Psychosenther 26:23-31 